\title{
A randomized controlled trial of long term effect of $B C M$ guided fluid management in MHD patients (BOCOMO study): rationales and study design
}

Li Liu ${ }^{1,2}$, Gang Long ${ }^{3}$, Jianwei Ren ${ }^{4}$, Jijun Li ${ }^{5}$, Jinsheng $X u^{6}$, Jinghong Lei ${ }^{7}$, Mao Li ${ }^{8}$, Moyan Qiu' ${ }^{9}$, Ping Yuan ${ }^{10}$, Weiming Sun ${ }^{11}$, Shan Lin ${ }^{12}$, Wenjun Liu' ${ }^{13}$, Yi Sun ${ }^{14}$, Yingchun Ma ${ }^{15}$, Yonghui Mao ${ }^{16}$, Yulan Shen ${ }^{17}$ and Li Zuo $0^{1,2^{*}}$

\begin{abstract}
Background: Bioimpedance analysis (BIA) has been reported as helpful in identifying hypervolemia. Observation data showed that hypervolemic maintenance hemodialysis (MHD) patients identified using BIA methods have higher mortality risk. However, it is not known if BIA-guided fluid management can improve MHD patients' survival. The objectives of the BOCOMO study are to evaluate the outcome of BIA guided fluid management compared with standard care.

Methods: This is a multicenter, prospective, randomized, controlled trial. More than 1300 participants from 16 clinical sites will be included in the study. The enrolment period will last 6 months, and minimum length of follow-up will be 36 months. MHD patients aged between 18 years and 80 years who have been on MHD for at least 3 months and meet eligibility criteria will be invited to participate in the study. Participants will be randomized to BIA arm or control arm in a 1:1 ratio. A portable whole body bioimpedance spectroscopy device (BCM_Fresenius Medical Care D GmbH) will be used for BIA measurement at baseline for both arms of the study. In the BIA arm, additional BCM measurements will be performed every 2 months. The primary intent-to-treat analysis will compare outcomes for a composite endpoint of death, acute myocardial infarction, stroke or incident peripheral arterial occlusive disease between groups. Secondary endpoints will include left ventricular wall thickness, blood pressure, medications, and incidence and length of hospitalization.

(Continued on next page)
\end{abstract}

\footnotetext{
* Correspondence: ZuoLi@bjmu.edu.cn

${ }^{1}$ Institute of Nephrology, Peking University First Hospital, Beijing, China

${ }^{2}$ Renal Division, Peking University First Hospital, Beijing, China

Full list of author information is available at the end of the article
} 
(Continued from previous page)

Discussions: Previous results regarding the benefit of strict fluid control are conflicting due to small sample sizes and unstable dry weight estimating methods. To our knowledge this is the first large-scale, multicentre, prospective, randomized controlled trial to assess whether BIS-guided volume management improves outcomes of MHD patients. The endpoints of the BOCOMO study are of utmost importance to health care providers. In order to obtain that aim, the study was designed with very careful important considerations related to the endpoints, sample size, inclusion criteria, exclusion criteria and so on. For example, annual mortality of Beijing MHD patients was around $10 \%$. To reach statistical significance, the sample size will be very large. By using composite endpoint, the sample size becomes reasonable and feasible. Limiting inclusion to patients with urine volume less than 800 $\mathrm{ml} /$ day the day before dialysis session will limit confounding due to residual renal function effects on the measured parameters. Patients who had received BIS measurement within 3 months prior to enrolment are excluded as data from such measurements might lead to protocol violation. Although not all patients enrolled will be incident patients, we will record the vintage of dialysis in the multivariable analysis.

Trial registration: Current Controlled Trials NCT01509937

Keywords: Hemodialysis, Bioimpedance, Dry weight, Body composition monitor, Randomized controlled trial

\section{Background}

Intra-dialytic symptoms are common among patients on maintenance hemodialysis (MHD). Adverse symptoms are associated with both hypervolemia and hypovolemia. Persistent hypervolemia causes hypertension, pulmonary edema, and congestive heart failure, and leads to higher mortality [1]. Compared with euvolemic patients, patients with recurrent episodes of intra-dialytic hypovolemia are at high risk of accelerated loss of residual renal function [2], vascular access function loss [3], brain atrophy [4], mesenteric infarction [5], and hence, higher morbidity and mortality $[6,7]$.

Patients with high pre-dialysis (pre-HD) fluid overload experience higher mortality risk compared with those with less pre-HD fluid burden $[1,8,9]$. It is an important strategy to reduce pre-HD fluid burden by restricting inter-dialysis weight gain and to achieve euvolemic (dry) weight post-HD by appropriate ultrafiltration. At present there is no objective gold standard method for estimating target post-HD dry weight and clinical examination is unreliable.

Several observational studies showed that strict postHD weight control was associated with better short term outcome [10] or long term survival [11], but other studies associated strict fluid control with increased morbidity and / or hospitalization [12,13]. These conflicting results regarding the relationship between fluid control and outcome mainly result from the lack of reliable method for the assessment of individual euvolemia with different studies used different definitions of dry weight including un-tolerated blood volume decrease or ultrafiltration limit [14].

Bioimpedance spectroscopy (BIS) had long been used to assess human body composition and has been validated by isotope dilution methods $[15,16]$ and reference body composition methods [16-18]. Recently, Wizemann et al. [1] used BIS to assess MHD patients' fluid status, and found that patients who had $15 \%$ or more expansion of extra-cellular fluid (ECF) suffered higher mortality risk compared with those had less than 15\% ECF expansion.

BIS appears to be a promising and a valuable tool in aiding dry weight estimation for MHD patients. Although detailed BIS-based dry weight estimating methods were published [10,19-21], and observational studies showed the potential benefits of BIS $[1,8]$, it is still not clear whether long-term outcome could be improved by regular dry weight adjustment according to BIS based method.

We designed this multicenter, prospective, randomized and controlled trial to explore the effect of BIS guided fluid management on long-term outcome in MHD patients.

\section{Methods}

\section{Study design}

This is an open label, multicenter, prospective, randomized controlled trial. The aim of the study is to assess if BIS guided fluid management can improve long term outcome in MHD patients. Body composition and hydration state will be assessed with a portable whole body bioimpedance spectroscopy device (BCM-Fresenius Medical Care D GmbH). The study will involve 16 clinical sites, enrolling more than 1300 participants. The enrolment period will last approximately 6 months, with a planned minimum 36 months follow-up period. Participants will be randomized to two arms, the control arm and $\mathrm{BCM}$ arm. Patients in both arms will receive BCM measurement, but results from the $\mathrm{BCM}$ measurement in the control group will be kept blinded to the investigators. Participants in control group will be followed up and their dry weights will be adjusted according to the dialysis center's standard clinical practice. Clinicians managing participants in the BCM group, in addition 
to routine practice, will receive $\mathrm{BCM}$ measurement and dry weight will be adjusted according to BCM output data estimating overhydration and dry weight. All of baseline demographics, clinical data, laboratory data, regular BCM measurement, and ultrasonic cardiograph (UCG) data will be recorded on study case report forms (CRFs).

The flow chart of the study is shown in Figure 1.

\section{Study objectives}

The objective of the study is to characterize the benefit of BCM guided fluid management in MHD patients.

\section{Primary objective}

The primary objective of the study is to compare incidence rate of a composite endpoint between BCM group and control group. Participants reach the primary endpoint if at least one of the following occurs: (1) death, (2) acute myocardial infarction, (3) cerebral thrombosis, (4) cerebral hemorrhage, (5) peripheral arterial occlusive disease. The diagnosis of endpoints will be made by the Endpoint Committee.

\section{Secondary objectives}

Secondary objectives are to compare the following indices between BCM arm and control arm: (1) left ventricular thickness and left ventricular ejection fraction, (2) Pre-HD and post-HD blood pressure, (3) antihypertensive medication, (4) hospitalization rate.

\section{Study protocol}

\section{Inclusion criteria}

Candidates must meet all of the following criteria to be eligible for enrollment into the study: (1) diagnosis of end stage renal disease (ESRD) and need for MHD, (2) age of 18 to 80 years old, (3) on MHD for at least 3 months, (4) dialysis frequency of at least 5 sessions per 2 weeks, not less than 4 hours per session and $\mathrm{Kt} / \mathrm{V}$ at least 1.2, (5) urine volume less than $800 \mathrm{~mL}$ per 24 hours the day before dialysis session, (6) BIS not used within recent 3 months, (7) dry weight regarded as adequate according to the patient's responsible doctor, (8) the ability to understand and willingness to sign an informed consent statement.

\section{Exclusion criteria}

Candidates meeting any one of the following criteria will not be eligible to be included in the study: (1) acute infection within prior month, (2) active rheumatic disease, or current receipt of cortical steroid or cytotoxic medication, (3) uncontrolled neoplasm, (4) acute myocardial infarction within prior month, (5) congestive heart failure (NYHA 3 - 4), (6) stroke within 3 prior months, (7) metallic prosthesis (contraceptive device, artificial joint), (8) amputation, (9) female of childbearing age who has a pregnancy plan, or is pregnant, or is breast feeding, (10) having a plan to reduce dialysis frequency, (11) having a renal transplantation plan or planning to transfer to peritoneal dialysis within 3 years, (12) participating or planning to participate in other clinical trials which would confound the current study.

Candidates meeting inclusion and exclusion criteria will be included in the study after providing informed consent. Study approval is obtained from the Institutional Review Board, Peking University First Hospital. This study is registered on ClinicalTrial.gov and has the identification number NCT 01509937.

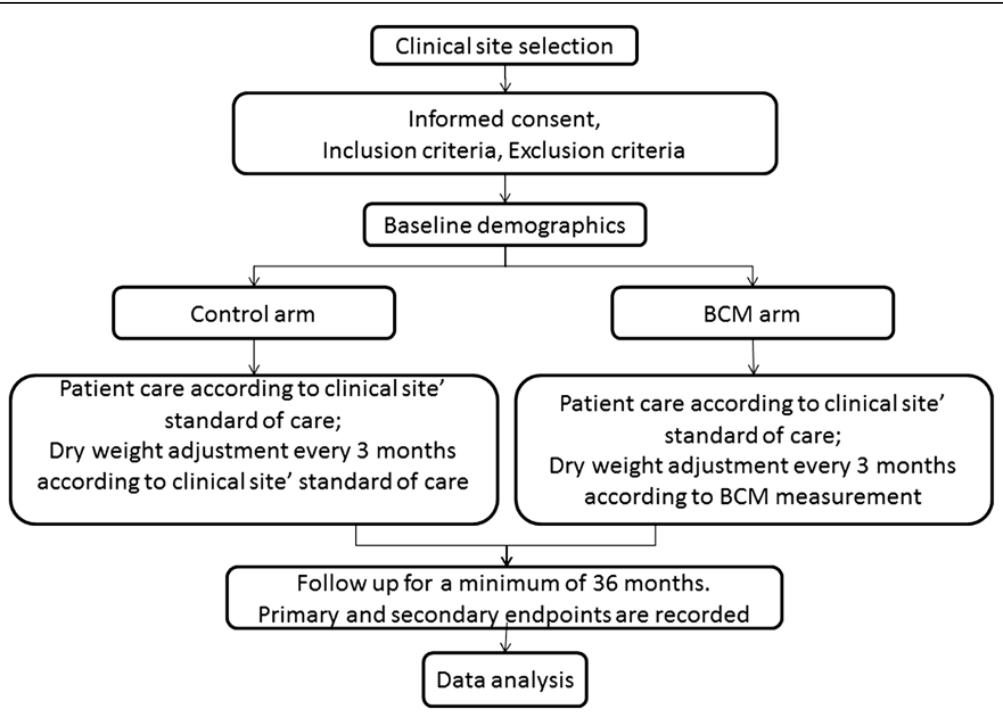

Figure 1 BOCOMO study Flow chart. 


\section{Data collection}

Summary of data collection is listed in Table 1.

Baseline data collection In brief, baseline data are collected before initiation of BCM monitoring and include demographics, ESRD treatment history, current vascular access type, detailed comorbid conditions assessment, current dialysis prescription and medications (oral, subcutaneous and intravenous), $\mathrm{CBC}$, routine laboratory data, Kt/V, iPTH and hsCRP. UCG will also be performed. BCM measurement will be done in participants in both BCM arm and control arm. In the control arm, BCM data will not be provided to patient care provider. 24 hours urine volume will be recorded the day before dialysis session. A physical examination will be performed.

Table 1 BOCOMO study schedule of data collection

\begin{tabular}{|c|c|c|}
\hline Item & $\begin{array}{l}\text { Baseline } \\
\text { frequency }\end{array}$ & Follow up frequency \\
\hline Demographic information & Once & - \\
\hline ESRD history $^{1}$ & Once & - \\
\hline Comorbidity $^{2}$ & Once & - \\
\hline Physical examination ${ }^{3}$ & Once & Every 2 months \\
\hline Timed urine collection ${ }^{4}$ & Once & Every 2 months \\
\hline $\mathrm{CBC}^{5}$ & Once & Every 3 months \\
\hline Routine laboratory data ${ }^{6}$ & Once & Every 3 months \\
\hline$\overline{\mathrm{Kt} / \mathrm{V}}$ & Once & Every 3 months \\
\hline$\overline{\mathrm{iPTH}}$ & Once & Every 3 months \\
\hline hsCRP & Once & Every 3 months \\
\hline UCG & Once & Every 12 months \\
\hline BCM measurement & Once & Every 2 months in BCM arm \\
\hline Dialysis prescription change $^{7}$ & Once & Throughout the study period \\
\hline Medication change ${ }^{8}$ & Once & Throughout the study period \\
\hline New clinical event ${ }^{9}$ & - & Throughout the study period \\
\hline
\end{tabular}

Abbreviation: BOCOMO study, body composition monitor study; ESRD, end stage renal disease; $C B C$, complete blood count; $\mathrm{Kt} / \mathrm{V}$, calculated using Daugirdas single pool equation; iPTH, intact parathyroid hormone; hsCRP, high sensitive C-reactive protein; UCG, ultrasonic cardiograph.

'ESRD history includes cause of ESRD, date of first dialysis, type of vascular access.

${ }^{2}$ Detailed history of comorbidity will be assessed, including vascular access events.

${ }^{3}$ Physical examination includes body weight, blood pressure, heart rate, edema, pulmonary rale, before and after hemodialysis session.

${ }^{4} 24$ hours urine volume collected the day before dialysis session will be recorded.

${ }^{5}$ Consists of hemoglobin, white blood cell count, platelet count.

${ }^{6}$ Consists of albumin, pre-Albumin, creatinine, urea, potassium, sodium, chloride, calcium, phosphate, bicarbonate, alkaline phosphatase (ALP), glucose, triglycerides, total cholesterol, low density lipoprotein, high density lipoprotein, brain natriuretic peptide (BNP).

${ }^{7}$ Any dialysis prescription change throughout the study period will be recorded.

${ }^{8}$ Any medication change throughout the study period will be recorded. ${ }^{9}$ Any new clinical event throughout the study period will be recorded, including hospitalization, new diagnosis, vascular access related event, treatment of abnormal laboratory value, dry weight adjustment.
Follow up data collection Physical examination and urine volume collection will be performed every 2 months. $\mathrm{CBC}$, routine laboratory data, and $\mathrm{Kt} / \mathrm{V}$ will be obtained every 3 months. iPTH and hsCRP will be measured every 3 months. UCG will be performed every 12 months.

BCM measurement will be performed every 2 months in $\mathrm{BCM}$ arm, data will be recorded and provided to the care provider.

\section{Dry Weight Adjustment Strategy}

All participants will be educated to reduce inter-dialysis weight gain to less than $5 \%$ body weight.

For participants in the control arm, dry weight will be adjusted according to the clinical site's standard clinical practice.

For participants in the BCM arm, dry weight will be adjusted according to the BCM estimates of overhydration and normovolemic weight. During each dialysis session, participants' dry weight will be increased or decreased toward BCM reported dry weight. The increment or decrement of dry weight will not be greater than $0.5 \mathrm{Kg}$ within a single dialysis session. Thus, in this arm participants' dry weights will be gradually adjusted to $\mathrm{BCM}$ reported value. If ultrafiltration is not tolerated because of hypovolemia based on symptoms and signs, such as muscle cramps, need for excessive saline, or symptomatic hypotension, adverse events will be recorded, and the actual dry weight will be adjusted by plus $0.5 \mathrm{Kg}$. If participants experience hypervolemic clinical episodes, such as hypertension related symptom, congestive heart failure, pulmonary edema, adverse events will also be recorded. If a patient experiences the same adverse effect of dry weight change for two consecutive visits, the patient will be removed from the study because of adverse event.

Dry weight adjustment flow chart in the BCM arm is shown in Figure 2.

\section{Clinical events}

Dialysis prescription change Dialysis prescription may be changed according to clinical site's standard practice including changes of dialyzer, blood flow rate, dialysate flow rate, frequency of dialysis, dialysis modality (conventional hemodialysis, high flux dialysis, hemofiltration, hemodiafiltration, and in combination of blood perfusion). Kt/V will remain not less than 1.2 despite dialysis prescription change. Dialysis prescription change is regarded as a clinical event and will be recorded.

Medications change Medications (oral, subcutaneous and intravenous drug administration) can be changed according to clinical site's practice, this including anti-hypertensives, active vitamin D and its analogues, erythrocyte stimulating 


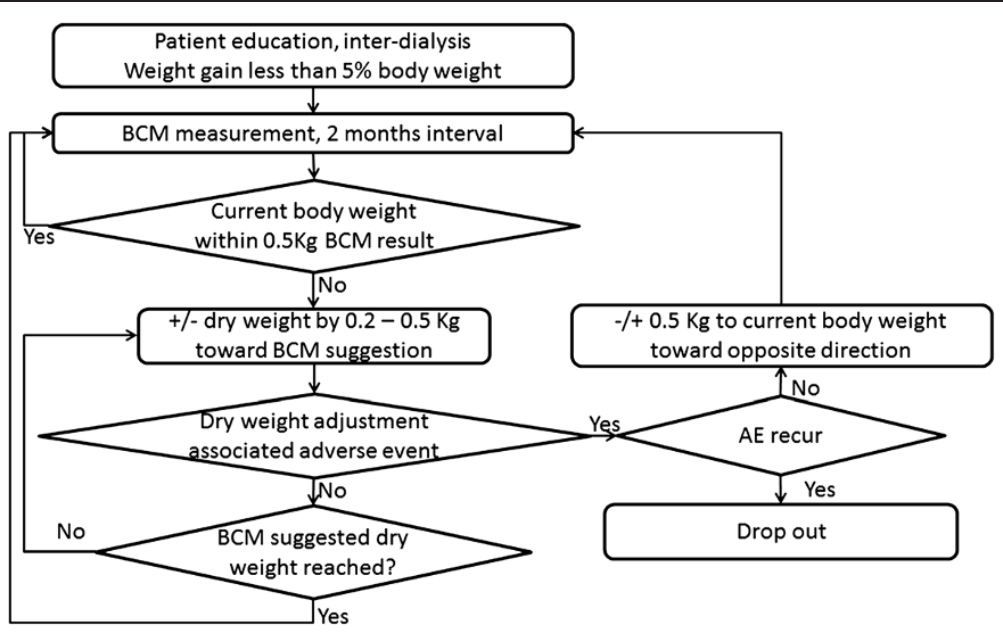

Figure 2 Dry weight adjustment flow chart in the BCM arm.

agents (ESA), and iron supplements. Medications change is regarded as a clinical event and will be recorded.

New diagnosis Any newly emerged diagnosis is regarded as a clinical event and will be recorded; hypotension and related clinical episode, hypertension and related clinical episode, pulmonary infection, diarrhea, angina pectoris, myocardial infarction, ischemic and hemorrhagic stroke, peripheral arterial occlusive disease, newly diagnosed neoplasm.

All deaths will be recorded as to date of death and cause.

Vascular access event Vascular access bleeding, thrombosis or malfunction will be recorded.

Dry weight change Each step of weight adjustment will be recorded.

Hospitalization Hospitalization will be recorded, including diagnosis leading to hospitalization, date of hospitalization, length of stay, and outcome.

Abnormal laboratory value If a procedure is performed to deal with abnormal laboratory value, the abnormal laboratory value is a clinical event and will be recorded.

Data censoring The following participants are censored: (1) transfer to peritoneal dialysis, (2) renal transplantation, (3) becomes pregnant, (4) receives metal implantation, or any other reasons that make BCM measurement impossible, (5) limb amputation, (6) withdrawal of consent, (7) receives any forms of BIS measurement other than those specified by the trial protocol, (8) relocates to other clinical site, (9) investigator requests the participant be withdrawn from the study.

\section{Safety issue}

The clinical use of BIS method had been approved by State of Food and Drug Administration (SFDA). As one form of BIS method, BCM device is widely used in Europe. BIS inject weak alternating current into human body, which cause no harm to human body.

\section{Statistical analysis \\ Study hypotheses}

This study is designed to test the primary hypotheses that patients receiving $\mathrm{BCM}$ guided fluid management have lower rate for the composite endpoint of mortality, cardiovascular and cerebrovascular events compared with patients receiving fluid management according to clinical site's standard care. The secondary hypotheses that will be tested are that patients receiving BCM measurement have (1) lower left ventricular thickness and higher left ventricular ejection fraction, (2) better blood pressure control, (3) less anti-hypertensive medication, (4) lower hospitalization rate.

\section{Sample size calculation}

Literature search failed to identify a study of similar design to this one. In an observational study by Wizemann et al. [1] of 269 MHD patients the patients were divided into hypervolemic group if their ECF was $15 \%$ higher than normal or normohydrated group if less than $15 \%$. The patients were followed for an average 3.5 years. There was $41 \%$ death in the hypervolemic group, and 30\% death in normohydrated group. Relative risk of death was $36.6 \%$ higher in hypervolemic group than in the other.

According to data from Beijing Hemodialysis Quality Control and Improvement Center, the annual mortality rate of Beijing MHD patients is around 10\%, it is estimated that 3 year mortality to be $30 \%$. It is also estimated that the rate of composite endpoint within 3 year 
period of time is $40 \%$. We make an assumption that BCM guided fluid management will reduce the 3-year composite endpoint rate from $40 \%$ to $32 \%$ (20\% relative risk reduction). To reach statistical significance with $\alpha<0.05$ and $1-\beta>80 \%$, the sample size required is 1128 . Allowing for a $20 \%$ loss-to-follow-up, the total sample size planned is 1354 .

\section{Analytical method}

Primary endpoint The primary analysis will be an intent-to-treat analysis comparing composite endpoint between the BCM and the control arms. The intent-totreat population is defined as all enrolled study participants. For this analysis, the follow-up time begins from the enrollment date to the composite endpoint, loss to follow-up, or end of study.

Multivariate Cox proportional hazards regression models will be used for analysis of the risk of death comparing BCM and control arms, with adjustment for age, sex, dialysis vintage, primary cause of ESRD, comorbid conditions, and other characteristics that may be associated with increased risk of death, such as inter-dialysis weight gain, hypertension and hsCRP.

Secondary endpoint The following 3 secondary endpoints will be compared between BCM and control arms using grouped t-test, and within group trend will also be analyzed: (1) left ventricular thickness and left ventricular ejection fraction, (2) pre-HD and post-HD blood pressure, (3) anti-hypertensive medication.

The secondary endpoint of incident rate of hospitalization will be compared between BCM arm and control arm using general Linear Model with Poisson regression.

Interim analysis The final analysis for primary endpoint and secondary analysis will be performed after completion of study.

Interim analysis is planned 1 year and 2 years after study initiation. The purpose of interim analysis includes: (1) adjustment of sample size according to collected real data, (2) early termination of study if statistically significance reached comparing primary endpoint between BCM arm and control arm, (3) early termination of study if incidence rate of fluid management related adverse events is significantly high in the BCM arm.

\section{Discussions}

\section{Strengths}

Previous results regarding the benefit of strict fluid control are conflicting due to small sample sizes and unstable dry weight estimating methods. To our knowledge this is the first large-scale, multicentre, prospective, randomized controlled trial to assess whether BIS-guided volume management improves outcomes of MHD patients. The endpoints of the BOCOMO study are of utmost importance to health care providers.

\section{About the design Inclusion and exclusion criteria}

(1) Only MHD patients who had been on MHD for at least 3 months are eligible. This is because patients with dialysis vintage less than 3 months are more likely to be hemodynamically unstable. Additionally, this criterion reduces the likelihood that pre-ESRD factors will be introduced into the study confounding the primary result. (2) Limiting inclusion to patients with urine volume less than $800 \mathrm{ml} /$ day the day before dialysis session will limit confounding due to residual renal function effects on the measured parameters. (3) Patients who had received BIS measurement within 3 months prior to enrolment are excluded as data from such measurements might lead to protocol violation.

\section{Changes of dialysis prescription and medications}

Fluid status change will definitely lead to physiological changes of the body. It is reasonable that some clinical parameter will change, like blood pressure, hemoglobin, hsCRP, BNP. So it is reasonable to allow dialysis prescription and medications change, which will serve as one of the secondary endpoints.

\section{Endpoint and sample size}

Annual mortality of Beijing MHD patients was around $10 \%$. To reach statistical significance, the sample size will be very large. By using composite endpoint, the sample size becomes reasonable and feasible.

\section{Dry weight adjustment in BCM arm}

"Real dry weight" is unknown in BOCOMO study. BCM reported dry weight might by significantly higher or lower than the real dry weight, which will lead to hypervolemia related acute symptoms and hypovolemia related acute symptoms, respectively. They will also worsen patients' long-term outcome. In these kinds of conditions, adverse events attributes to BCM arm will be recorded.

Another issue regarding dry weight adjustment in $\mathrm{BCM}$ arm is the amount of each dry weight increase or decrease. Large amount of dry weight change is not allowed because significant dry weight increase or decrease during one HD session will lead to dramatic blood volume increase or decrease during HD session and associated clinical manifestation.

\section{Limitations}

There are several limitations of the BOCOMO study: (1) using composite endpoint instead of mortality, (2) inclusion 
of prevalent patients instead of incident patients (but dialysis vintage will be included in multivariable analysis), (3) most clinical sites are located in Beijing.

\section{Conclusion}

In summary, the BOCOMO study has the potential to be the largest and the first prospective randomized controlled trial to evaluate the long term clinical benefit of BIS-guided fluid management in MHD patients. The aim of the study is to validate whether BIS based dry weight adjustment decrease incidence composite endpoint of death, acute myocardial infarction, stroke, and peripheral arterial disease. The result will be of great clinical importance.

\section{Competing interests}

The authors declare that they have no competing interests.

\section{Authors' contributions}

All authors participated in the design and discussion of the protocol. All authors read and approved the final manuscript.

\section{Acknowledgements}

This work was supported by a grant from the Fresenius Medical Care Shanghai Co. LTD. None of the authors has a conflict of interest to declare associated with this review.

\section{Author details}

${ }^{1}$ Institute of Nephrology, Peking University First Hospital, Beijing, China. ${ }^{2}$ Renal Division, Peking University First Hospital, Beijing, China. ${ }^{3}$ Renal Department, Tianjin Union Medicine Center, Tianjin, China. ${ }^{4}$ Renal Department, Aviation General Hospital, Beijing, China. ${ }^{5}$ Renal Department, PLA General Hospital First Hospital, Beijing, China. ${ }^{6}$ Renal Department, Hebe Medical University Forth Hospital, Hebei, China. ${ }^{7}$ Renal Department, Beijing Aerospace General Hospital, Beijing, China. ${ }^{8}$ Renal Department, Beijing Puren Hospital, Beijing, China. ${ }^{9}$ Renal Department, Beijing Wangjing Hospital, Beijing, China. ${ }^{10}$ Renal Department, Tianjin Third Central Hospital, Tianjin, China. ${ }^{11}$ Renal Department, Beijing Shijitan Hospital, Beijing, China. ${ }^{12}$ Renal Department, Tianjin Medical University General Hospital, Tianjin, China. ${ }^{13}$ Renal Department, Guanganmen Hospital, China Academy of Chinese Medical Sciences, Guanganmen, China. ${ }^{14}$ Renal Department, Capital University Fuxing Hospital, Beijing, China. ${ }^{15}$ Renal Department, China Rehabilitation Research Center, Beijing Boai Hospital, Beijing, China. ${ }^{16}$ Renal Department, Beijing Hospital of Ministry of Health, Beijing, China. ${ }^{17}$ Renal Department, Miyun Hospital, Beijing, China.

Received: 11 September 2012 Accepted: 14 September 2012 Published: 25 September 2012

\section{References}

1. Wizemann V, Wabel P, Chamney P, Zaluska W, Moissl U, Rode C, MaleckaMasalska T, Marcelli D: The mortality risk of overhydration in haemodialysis patients. Nephrol Dial Transplant. 2009, 24(5):1574-1579. England.

2. Jansen MA, Hart AA, Korevaar JC, Dekker FW, Boeschoten EW, Krediet RT: Predictors of the rate of decline of residual renal function in incident dialysis patients. Kidney Int. 2002, 62(3):1046-1053. United States.

3. Puskar D, Pasini J, Savic I, Bedalov G, Sonicki Z: Survival of primary arteriovenous fistula in 463 patients on chronic hemodialysis. Croat Med J. 2002, 43(3):306-311. Croatia.

4. Mizumasa T, Hirakata H, Yoshimitsu T, Hirakata E, Kubo M, Kashiwagi M, Tanaka H, Kanai H, Fujimi S, lida M: Dialysis-related hypotension as a cause of progressive frontal lobe atrophy in chronic hemodialysis patients: a 3year prospective study. Nephron Clin Pract 2004, 97(1):c23-c30. Switzerland.

5. John AS, Tuerff SD, Kerstein MD: Nonocclusive mesenteric infarction in hemodialysis patients. J Am Coll Surg 2000, 190(1):84-88. UNITED STATES.
6. Shoji T, Tsubakihara Y, Fujii M, Imai E: Hemodialysis-associated hypotension as an independent risk factor for two-year mortality in hemodialysis patients. Kidney Int 2004, 66(3):1212-1220. United States.

7. Tisler A, Akocsi K, Harshegyi I, Varga G, Ferenczi S, Grosz M, Kulcsar I, Locsey L, Samik J, Solt I, Szegedi J, Toth E, Wagner G, Kiss I: Comparison of dialysis and clinical characteristics of patients with frequent and occasional hemodialysis-associated hypotension. Kidney Blood Press Res 2002, 25(2):97-102. Switzerland.

8. Pillon L, Piccoli A, Lowrie EG, Lazarus JM, Chertow GM: Vector length as a proxy for the adequacy of ultrafiltration in hemodialysis. Kidney Int 2004, 66(3):1266-1271. United States.

9. Movilli E, Gaggia P, Zubani R, Camerini C, Vizzardi V, Parrinello G, Savoldi S, Fischer MS, Londrino F, Cancarini G: Association between high ultrafiltration rates and mortality in uraemic patients on regular haemodialysis. A 5-year prospective observational multicentre study. Nephrol Dial Transplant 2007, 22(12):3547-3552. England.

10. Chamney PW, Kramer M, Rode C, Kleinekofort W, Wizemann V: A new technique for establishing dry weight in hemodialysis patients via whole body bioimpedance. Kidney Int 2002, 61(6):2250-2258. United States.

11. Ozkahya M, Ok E, Toz H, Asci G, Duman S, Basci A, Kose T, Dorhout MEJ: Long-term survival rates in haemodialysis patients treated with strict volume control. Nephrol Dial Transplant 2006, 21(12):3506-3513. England.

12. Reddan DN, Szczech LA, Hasselblad V, Lowrie EG, Lindsay RM, Himmelfarb J, Toto RD, Stivelman J, Winchester JF, Zillman LA, Califf RM, Owen WF Jr: Intradialytic blood volume monitoring in ambulatory hemodialysis patients: a randomized trial. J Am Soc Nephrol 2005, 16(7):2162-2169. United States.

13. Agarwal R, Alborzi P, Satyan S, Light RP: Dry-weight reduction in hypertensive hemodialysis patients (DRIP): a randomized, controlled trial. Hypertension 2009, 53(3):500-507. United States.

14. Charra B: Fluid balance, dry weight, and blood pressure in dialysis. Hemodial Int 2007, 11(1):21-31. Canada.

15. Moiss UM, Wabel P, Chamney PW, Bosaeus I, Levin NW, Bosy-Westphal A, Korth O, Muller MJ, Ellegard L, Malmros V, Kaitwatcharachai C, Kuhlmann MK, Zhu F, Fuller NJ: Body fluid volume determination via body composition spectroscopy in health and disease. Physiol Meas. 2006, 27(9):921-933. England.

16. den Ham ECv, Kooman JP, Christiaans MH, Nieman FH, Van Kreel BK, Heidendal GA, Van Hooff JP: Body composition in renal transplant patients: bioimpedance analysis compared to isotope dilution, dual energy X-ray absorptiometry, and anthropometry. J Am Soc Nephrol 1999, 10(5):1067-1079. UNITED STATES.

17. McClanahan BS, Stockton MB, Lanctot JQ, Relyea G, Klesges RC, Slawson DL, Schilling LP: Measurement of body composition in 8-10-year-old African-American girls: a comparison of dual-energy X-ray absorptiometry and foot-to-foot bioimpedance methods. Int J Pediatr Obes 2009, 4(4):389-396. England.

18. Lintsi M, Kaarma H, Kull I: Comparison of hand-to-hand bioimpedance and anthropometry equations versus dual-energy $\mathrm{X}$-ray absorptiometry for the assessment of body fat percentage in 17-18-year-old conscripts. Clin Physiol Funct Imaging. 2004, 24(2):85-90

19. Kraemer M: A new model for the determination of fluid status and body composition from bioimpedance measurements. Physiol Meas 2006, 27(9):901-919. England.

20. Dou YN, Cheng XY, Liu L, Bai XF, Wu LY, Guo WY, Zhao XJ, Wang F, Cao LY, Zuo L: Development and Validation of a New Dry Weight Estimation Method Using Single Frequency Bioimpedance in Hemodialysis Patients. Blood Purification 2011, 32:278-285.

21. Guida B, De Nicola L, Trio R, Pecoraro P, lodice C, Memoli B: Comparison of vector and conventional bioelectrical impedance analysis in the optimal dry weight prescription in hemodialysis. Am J Nephrol 2000, 20(4):311-318. SWITZERLAND.

doi:10.1186/1471-2369-13-120

Cite this article as: Liu et al: A randomized controlled trial of long term effect of BCM guided fluid management in MHD patients (BOCOMO study): rationales and study design. BMC Nephrology 2012 13:120. 